Case Report

\title{
Spinal Cord Infarction in the Course of a Septic Shock: About One Case and Review of the Literature
}

\author{
P. Henin, A. Molderez, V. Huberlant, and H. Trine \\ Groupe Jolimont, Centre Hospitalier de Jolimont, 159 rue Ferrer, 7100 Haine Saint Paul, Belgium \\ Correspondence should be addressed to V. Huberlant; vhuberlant@hotmail.com
}

Received 23 November 2016; Accepted 24 January 2017; Published 20 February 2017

Academic Editor: Kurt Lenz

Copyright (C) 2017 P. Henin et al. This is an open access article distributed under the Creative Commons Attribution License, which permits unrestricted use, distribution, and reproduction in any medium, provided the original work is properly cited.

We report the case of a patient admitted to our intensive care unit in the course of a septic shock, secondary to cholangitis. After rapid hemodynamic stabilization, antibiotherapy, and endoscopic extraction of bile ducts stones, she appeared to have developed flaccid paraplegia. The suspected diagnosis of medullar ischemia was confirmed by typical MRI findings. This case stresses the potential pathogenic role of hypotension in medullar ischemia and the place of magnetic resonance imaging (MRI) as a reliable diagnostic tool.

\section{Introduction}

Medullar ischemia is a rare and severe condition [1] that can lead to death or persistent paraplegia [2-4].

Its most usual etiologies are thoracoabdominal aortic surgery, trauma, or cardiovascular diseases. It can also be due to deep hypotension and global ischemia [4-6].

We report a seldom case of medullar ischemia occurring in the course of a septic shock. We also describe the typical magnetic resonance imaging (MRI) findings, most accurate tool for diagnosis confirmation $[1,3,4,7-10]$.

\section{Case Report}

A 55-year-old Caucasian woman was admitted through the Emergency Room with severe abdominal pain and dehydration. Her main past medical history included diabetes, active alcoholism, smoking, chronic pancreatitis, and hypercholesterolemia. She had a recent episode of acute on chronic alcoholic pancreatitis, with transient acute renal failure. She was transferred to our intensive care unit in septic shock with severe hypotension (MAP $<40 \mathrm{mmHg}$ at the time of admission) and transient sinus bradycardia.

She also had fever, clinical jaundice, and peripheral signs of hypoperfusion. The neurological exam showed impaired consciousness but no motor or sensitive deficit.
Laboratory data showed lactic metabolic acidosis, acute renal failure, and cholestasis. There was no coagulation disorder.

An abdominal CT scan showed hepatic steatosis, pancreatic calcifications, gallbladder hydrops and lithiasis, and enlargement of the extrahepatic bile ducts. Ultrasonography confirmed the suspected diagnosis of cholangitis: enlargement of the choledochus $(12 \mathrm{~mm})$, occluded in its distal part by a $9 \mathrm{~mm}$ lithiasis.

Within the first hour, the MAP was restored to normal range $(>65 \mathrm{mmHg})$. She received empirical antibiotherapy (Amoxicillin-Clavulanate) and an urgent ERCP was performed, with successful extraction of the common bile duct stone.

On the next day, her clinical exam confirmed the recovery of stable hemodynamic parameters, with normal urine output. Lactic acidosis was corrected, and renal function and liver enzymes were improving.

Unfortunately, the patient appeared to have developed complete flaccid paraplegia. A thorough neurological exam showed a sensory (pain and temperature) and motor deficit at T10 level and loss of sphincter control. We suspected spinal cord ischemia.

Somatosensory evoked potentials were compatible with that diagnosis. 


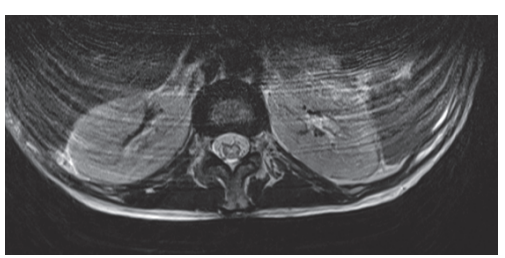

(a)

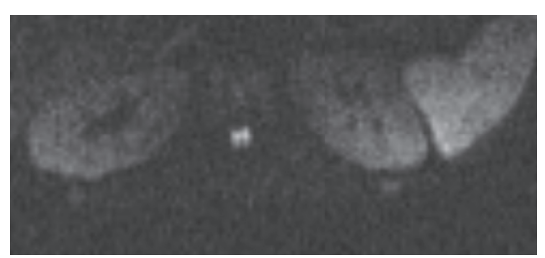

(b)

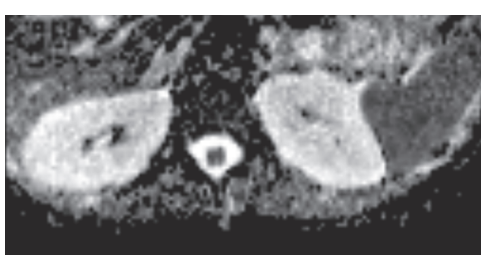

(c)

Figure 1: (a) The sagittal T2-weighted image shows a hyperintense lesion of the conus medullaris. (b) High signal is observed at the corresponding level on axial DWI. (c) Low ADC value on the ADC map confirmed the hypothesis of spinal cord infarction.

MRI demonstrated on the sagittal T2-weighted image a hyperintense lesion of the conus medullaris and high signal was observed at the corresponding level on axial DWI (Diffusion Weighted Imaging); low ADC (Apparent Diffusion Coefficient) value on the ADC map confirmed the hypothesis of spinal cord infarction (Figure 1).

The patient remained stable and further recovered from all her biological disorders. Bacteriology remained negative and antibiotics were discontinued on day 5 .

Unfortunately, apart from discrete contraction of the proximal muscles of the right leg, the patient did not recover from her neurologic disorder and had persistent flaccid paraplegia, abolition of temperature and pain sensitivity, and sphincter dysfunction, despite thorough multidisciplinary rehabilitation.

She died a few months later in the rehabilitation center, in the course of an acute myocardial infarct.

\section{Discussion}

Acute onset of paraplegia is rarely seen in the ICU setting. The differential diagnosis will mainly be orientated by clinical context: recent history of trauma, lumbar or epidural puncture, recent aortic surgery, recent history of hemodynamic instability (shock or global hypoperfusion), cardiovascular comorbidities, especially with thromboembolic events, anticoagulant therapies, systemic inflammatory diseases, or cancers.

We can distinguish extramedullar and medullar etiologies.

Extramedullar etiologies include polyradiculitis (Guillain-Barre syndrome) [11] or can be part of the clinical manifestations of ion disorders [12] like hyperkaliemia or hypercalcemia. Their onset will usually be more subacute. Psychogenic disorders can also mimic paraplegia [13].

Medullar etiologies are more relevant to our discussion. They can be traumatic: vertebral trauma with cord lesion [14], through mechanisms of transection, compression, contusion, or vascular compromise, and spinal epidural bleeding after puncture [15].

Medullar etiologies can also be nontraumatic: medullar ischemia or thromboembolism, as discussed in this case report, spinal bleeding, spontaneous [16] or under anticoagulant therapy [17], tumor, or abscess compression $[18,19]$.

Other nontraumatic medullar etiologies of paraplegia can be seen, but their course is usually more subacute, not as sudden as in our case, and will often be part of a more complex clinical situation: hepatic myelopathy in liver cirrhosis [20], paraneoplastic necrotizing myelopathy [21], systemic inflammatory diseases like multiple sclerosis or sarcoidosis [22-24], or infectious diseases like viral or bacterial myelitis (e.g., Lyme's disease or CMV). Some (around 15\%) cases of transverse myelitis remain unclear and are referred to as idiopathic [25].

Medullar ischemia is a rare diagnosis [1]. Its incidence is difficult to evaluate and few series are published $[1-7,9$, 10, 26-28]. Anterior spinal artery syndrome (ASAS) is the most usual clinical presentation [1]. This syndrome results from an infarction of the anterior two-thirds of the spinal cord. The midthoracic level is usually seen as the watershed zone for ischemic vulnerability, but some authors argue that lower (lumbar or lumbosacral) levels seem most vulnerable to global ischemic events $[4,29]$.

The common symptoms are sudden weakness under the level of ischemia, flaccid paraplegia, areflexia, urinary bladder and anal sphincter dysfunction, and loss of pain and temperature perception without proprioceptive disorder (no involvement of the posterior third of the spinal cord) [4]. The onset is usually sudden, with maximal impairment often reached within the first hour [10]. Severity of impairment can be defined using the American Spinal Injury Association (ASIA) scoring [8] as follows:

(1) Complete: no motor or sensory function is found in the lowest sacral segment (S4-S5).

(2) Incomplete: sensory function is found below neurologic level and in S4-S5; no motor function is found below neurologic level.

(3) Incomplete: motor function is preserved below neurologic level and more than half of the key muscle groups below neurologic level have a muscle grade less than 3.

(4) Incomplete: motor function is preserved below neurologic level and at least half of the key muscle groups below neurologic level have a muscle grade greater than 3.

(5) Normal: sensory and motor function is normal.

Initial impairment can be mild (ASIA scoring $\mathrm{C}$ or D), in about 40 to $50 \%$ of all cases, moderate (ASIA B), or severe (ASIA A), each around 25\% $[5,10]$. 
Etiologies of ASAS are multiple. The most common causes are surgery of the thoracoabdominal aorta, trauma, all vascular disorders like thromboembolism and inflammatory diseases, and global hypoxic-ischemic events such as cardiac arrest or severe hypotension [4, 9, 10]. Cardiovascular risk factors such as hypertension are common among patients with ASAS $[2,5,7]$.

The best diagnostic tool for ASAS is neuroimaging with MRI. The typical findings are focal cord swelling with increased T2 signal in the central part of the spinal cord and in DWI (Diffusion Weighted Imaging), an increased medullar signal associated with a low ADC (Apparent Diffusion Coefficient) value $[6,10-12,30]$.

The prognosis of ASAS is variable and can range from full recovery to long term persistence of complete paraplegia, loss of sensitivity, sphincter dysfunction, and chronic pain. The percentage of different outcomes is difficult to evaluate, depending on the author and because of the relatively small size of published series [1-7, 9, 10, 26-28, 31]. Death occurs in less than $10 \%[1,5,6]$ to more than $20 \%$ [9] of cases. Complete or satisfactory (ambulatory) recovery seems to occur in $25 \%$ of the survivors according to the pessimistic [9] and up to more than $50 \%$ according to the optimistic authors $[1,2]$. Complete persistent impairment (wheelchair dependence, bladder catheter, loss of sensitivity, and chronic pain) also ranges from 20 [5] to more than 50\% [9], depending on authors. The remaining patients will have partial recovery and a mix of motor, sensory, and autonomic sequelae.

Recovery occurs mainly in the first 2 to 4 weeks [4], but meaningful improvement can occur after several months in a significant minority of cases [10].

All authors agree that severity of the initial impairment is associated with a poor outcome $[2,4,6,9,10]$. The correlation between age at onset and outcome is disputed. Some authors find a better prognosis in younger patients [10], while others describe a poorer prognosis below age of 55 years [2].

Severe hypotension is a recognized etiological factor of spinal cord ischemia $[29,32]$, and our case shows the potential deleterious consequences of sepsis related shock on medullar perfusion. It stresses the importance of quickly restoring a MAP above $65 \mathrm{mmHg}$ according to the surviving sepsis campaign guidelines [33], especially in patients with cardiovascular risk factors, in order to prevent, among other complications, medullar ischemia.

There is no specific therapy for ASAS. Treatment in the acute phase is thus mainly supportive. Selective injection of Dexamethasone and Urokinase in the Adamkiewicz artery (arteria radicularis magna) has been proposed in a small group of 3 patients [28].

During thoracoabdominal aortic surgery, lumbar CSF drainage can be used to protect medullar perfusion. The rationale is to maintain a spinal perfusion pressure (MAP intrathecal pressure) above 70 to $80 \mathrm{mmHg}$, by supporting hemodynamics and draining CSF to maintain intrathecal pressure below $10 \mathrm{mmHg}$ [34].

Other techniques are proposed in order to reduce the incidence of perioperative medullar ischemia, such as perioperative somatosensory evoked potentials [35], induced hypothermia [36], intrathecal papaverine [37], or epidural cooling [38].

\section{Conclusion}

Spinal ischemia is a severe condition. Its most common presentation is ASAS. It is a well known complication of thoracoabdominal surgery but can also result from traumatic, cardiovascular, or systemic disorders and from severe hypotension and global ischemia.

Its onset is sudden, and its prognosis is variable, from recovery to persistent paraplegia or death.

A severe initial impairment is related to a poorer prognosis, but recovery can occur even in severe cases, and delayed recoveries are not exceptional.

This case report shows the potential consequences of septic shock on medullar perfusion and stresses the importance of rapidly restoring MAP, according to the surviving sepsis campaign.

It also shows the key diagnostic role of MRI and its typical findings in cases of ASAS.

\section{Competing Interests}

The authors declare no conflict of interests.

\section{References}

[1] T. A. Sandson and J. H. Friedman, "Spinal cord infarction. Report of 8 cases and review of the literature," Medicine (Baltimore), vol. 68, no. 5, pp. 282-292, 1989.

[2] K. Nedeltchev, T. J. Loher, F. Stepper et al., "Long-term outcome of acute spinal cord ischemia syndrome," Stroke, vol. 35, no. 2, pp. 560-565, 2004.

[3] C. Masson, J. P. Pruvo, J. F. Meder et al., "Spinal cord infarction: clinical and magnetic resonance imaging findings and short term outcome," Journal of Neurology, Neurosurgery and Psychiatry, vol. 75, no. 10, pp. 1431-1435, 2004.

[4] S. Salvador de la Barrera, A. Barca-Buyo, A. Montoto-Marqués, M. E. Ferreiro-Velasco, M. Cidoncha-Dans, and A. RodriguezSotillo, "Spinal cord infarction: prognosis and recovery in a series of 36 patients," Spinal Cord, vol. 39, no. 10, pp. 520-525, 2001.

[5] W. P. Cheshire, C. C. Santos, E. W. Massey, and J. F. Howard Jr., "Spinal cord infarction: etiology and outcome," Neurology, vol. 47, no. 2, pp. 321-330, 1996.

[6] C. E. Robertson, R. D. Brown Jr., E. F. M. Wijdicks, and A. A. Rabinstein, "Recovery after spinal cord infarcts: long-term outcome in 115 patients," Neurology, vol. 78, no. 2, pp. 114-121, 2012.

[7] S. Weidauer, M. Nichtweiss, H. Lanfermann, and F. E. Zanella, "Spinal cord infarction: MR imaging and clinical features in 16 cases," Neuroradiology, vol. 44, no. 10, pp. 851-857, 2002.

[8] American Spinal Injury Association, International Standards for Neurological Classification of Spinal Cord Injury, American Spinal Injury Association, Chicago, Ill, USA, 2002.

[9] M.-Y. Cheng, R.-K. Lyu, Y.-J. Chang et al., "Spinal cord infarction in Chinese patients: clinical features, risk factors, imaging and prognosis," Cerebrovascular Diseases, vol. 26, no. 5, pp. 502$508,2008$. 
[10] J. Novy, A. Carruzzo, P. Maeder, and J. Bogousslavsky, “Spinal cord ischemia: clinical and imaging patterns, pathogenesis, and outcomes in 27 patients," Archives of Neurology, vol. 63, no. 8, pp. 1113-1120, 2006.

[11] T. Solomon and H. Willison, "Infectious causes of acute flaccid paralysis," Current Opinion in Infectious Diseases, vol. 16, no. 5, pp. 375-381, 2003.

[12] S. Evers, A. Engelien, V. Karsch, and M. Hund, "Secondary hyperkalaemic paralysis," Journal of Neurology Neurosurgery and Psychiatry, vol. 64, no. 2, pp. 249-252, 1998.

[13] J. Stone, C. Warlow, and M. Sharpe, "The symptom of functional weakness: a controlled study of 107 patients," Brain, vol. 133, no. 5, pp. 1537-1551, 2010.

[14] J. R. Chapman and P. A. Anderson, "Thoracolumbar spine fractures with neurologic deficit," Orthopedic Clinics of North America, vol. 25, no. 4, pp. 595-612, 1994.

[15] M. T. Pitkänen, U. Aromaa, D. A. Cozanitis, and J. G. Förster, "Serious complications associated with spinal and epidural anaesthesia in Finland from 2000 to 2009," Acta Anaesthesiologica Scandinavica, vol. 57, no. 5, pp. 553-564, 2013.

[16] R. H. Thiele, Z. A. Hage, D. L. Surdell, S. L. Ondra, H. H. Batjer, and B. R. Bendok, "Spontaneous spinal epidural hematoma of unknown etiology: case report and literature review," Neurocritical Care, vol. 9, no. 2, pp. 242-246, 2008.

[17] J. Furlan, G. W. Hawryluk, J. Austin, and M. G. Fehlings, "Spinal haemorrhage during anticoagulant regimen for thromboprophylaxis: a unique form of central nervous system haemorrhage," Journal of Neurology, Neurosurgery and Psychiatry, vol. 83, no. 7, pp. 746-752, 2012.

[18] R. J. Sevick and C. J. Wallace, "MR imaging of neoplasms of the lumbar spine," Magnetic Resonance Imaging Clinics of North America, vol. 7, no. 3, pp. 539-553, 1999.

[19] R. O. Darouiche, "Spinal epidural abscess," New England Journal of Medicine, vol. 355, no. 19, pp. 2012-2020, 2006.

[20] R. Nardone, T. Buratti, A. Oliviero, A. Lochmann, and F. Tezzon, "Corticospinal involvement in patients with a portosystemic shunt due to liver cirrhosis: A MEP Study," Journal of Neurology, vol. 253, no. 1, pp. 81-85, 2006.

[21] V. J. Ojeda, "Necrotizing myelopathy associated with malignancy. A clinicopathologic study of two cases and literature review," Cancer, vol. 53, no. 5, pp. 1115-1123, 1984.

[22] R. Bakshi, P. R. Kinkel, L. L. Mechtler et al., "Magnetic resonance imaging findings in 22 cases of myelitis: comparison between patients with and without multiple sclerosis," European Journal of Neurology, vol. 5, no. 1, pp. 35-48, 1998.

[23] M. Harzheim, U. Schlegel, H. Urbach, T. Klockgether, and S. Schmidt, "Discriminatory features of acute transverse myelitis: a retrospective analysis of 45 patients," Journal of the Neurological Sciences, vol. 217, no. 2, pp. 217-223, 2004.

[24] S. Saleh, C. Saw, K. Marzouk, and O. Sharma, "Sarcoidosis of the spinal cord: literature review and report of eight cases," Journal of the National Medical Association, vol. 98, no. 6, pp. 965-974, 2006.

[25] J. De Seze, C. Lanctin, C. Lebrun et al., "Idiopathic acute transverse myelitis: application of the recent diagnostic criteria," Neurology, vol. 65, no. 12, pp. 1950-1953, 2005.

[26] M. Shinoyama, T. Takahashi, H. Shimizu, T. Tominaga, and M. Suzuki, "Spinal cord infarction demonstrated by diffusionweighted magnetic resonance imaging," Journal of Clinical Neuroscience, vol. 12, no. 4, pp. 466-468, 2005.
[27] R. G. Nogueira, R. Ferreira, P. E. Grant et al., "Restricted diffusion in spinal cord infarction demonstrated by magnetic resonance line scan diffusion imaging," Stroke, vol. 43, no. 2, pp. 532-535, 2012.

[28] H. Baba, K. Tomita, T. Kawagishi, and S. Imura, "Anterior spinal artery syndrome," International Orthopaedics, vol. 17, no. 6, pp. 353-356, 1993.

[29] N. Duggal and B. Lach, "Selective vulnerability of the lumbosacral spinal cord after cardiac arrest and hypotension," Stroke, vol. 33, no. 1, pp. 116-121, 2002.

[30] M. M. Thurnher and R. Bammer, "Diffusion-weighted MR imaging (DWI) in spinal cord ischemia," Neuroradiology, vol. 48, no. 11, pp. 795-801, 2006.

[31] E. Iseli, A. Cavigelli, V. Dietz, and A. Curt, "Prognosis and recovery in ischaemic and traumatic spinal cord injury: clinical and electrophysiological evaluation," Journal of Neurology Neurosurgery and Psychiatry, vol. 67, no. 5, pp. 567-571, 1999.

[32] C.-C. Lin, S.-Y. Chen, C. Lan, T. Ting-Fang Shih, M.-C. Lin, and J.-S. Lai, "Spinal cord infarction caused by cardiac tamponade," American Journal of Physical Medicine and Rehabilitation, vol. 81, no. 1, pp. 68-71, 2002.

[33] R. P. Dellinger, M. M. Levy, A. Rhodes et al., "Surviving sepsis campaign: international guidelines for management of severe sepsis and septic shock: 2012," Critical Care Medicine, vol. 41, no. 2, pp. 580-637, 2013.

[34] H. Takayama and M. A. Borger, "Preventing spinal cord injury during thoracic aortic surgery: simpler than we thought?" Journal of Thoracic and Cardiovascular Surgery, vol. 149, no. 1, pp. 366-368, 2015.

[35] J. D. Galla, M. A. Ergin, S. L. Lansman et al., "Use of somatosensory evoked potentials for thoracic and thoracoabdominal aortic resections," Annals of Thoracic Surgery, vol. 67, no. 6, pp. 1947-1952, 1999.

[36] G. Di Luozzo, "Visceral and spinal cord protection during thoracoabdominal aortic aneurysm repair: clinical and laboratory update," Journal of Thoracic and Cardiovascular Surgery, vol. 145, no. 3S, pp. S135-S138, 2013.

[37] B. Lima, E. R. Nowicki, E. H. Blackstone et al., "Spinal cord protective strategies during descending and thoracoabdominal aortic aneurysm repair in the modern era: the role of intrathecal papaverine," Journal of Thoracic and Cardiovascular Surgery, vol. 143, no. 4, pp. 945-952.el, 2012.

[38] R. P. Cambria, J. K. Davison, C. Carter et al., "Epidural cooling for spinal cord protection during thoracoabdominal aneurysm repair: a five-year experience," Journal of Vascular Surgery, vol. 31, no. 6, pp. 1093-1102, 2000. 


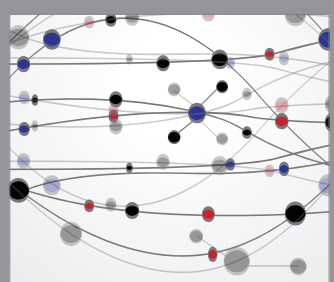

The Scientific World Journal
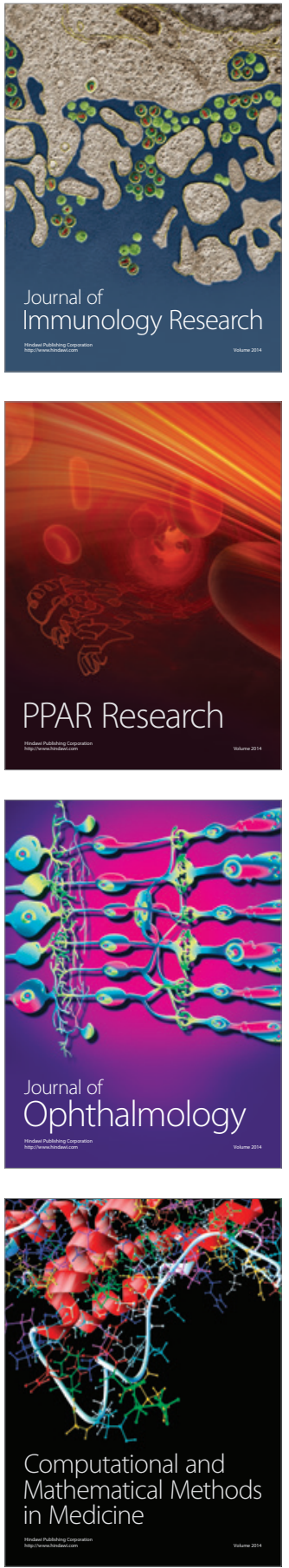

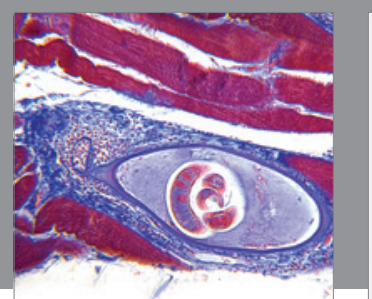

Gastroenterology Research and Practice
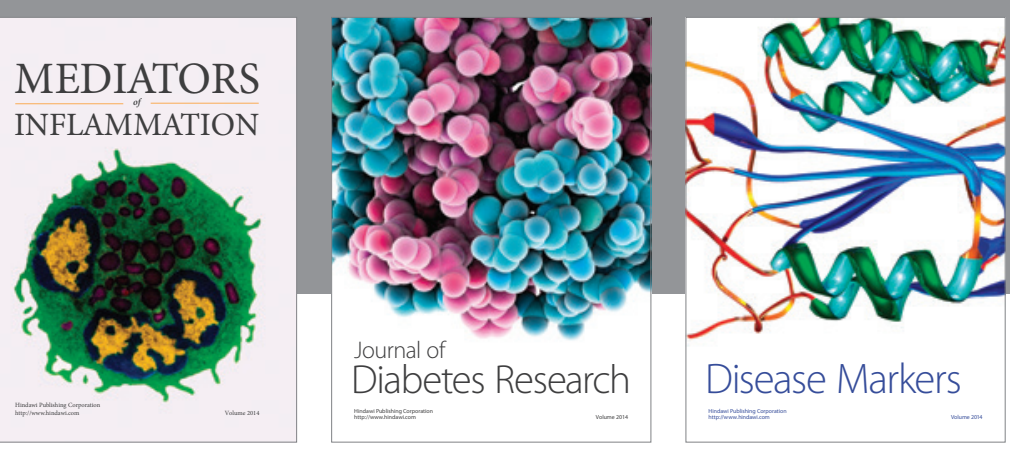

Disease Markers

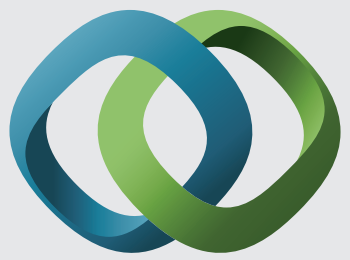

\section{Hindawi}

Submit your manuscripts at

https://www.hindawi.com
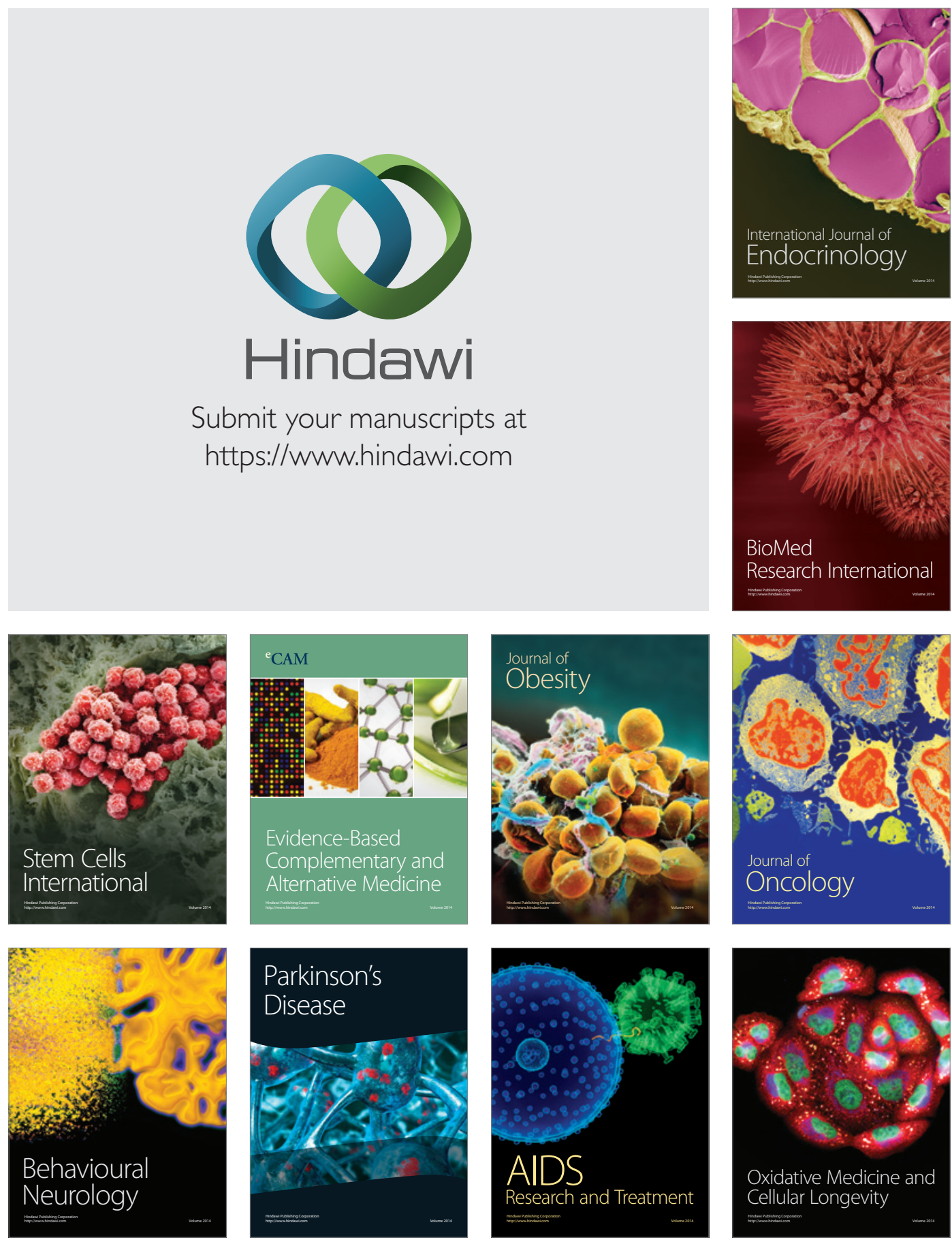